\title{
Dislocation Model for Restacking Phase Transitions in Crystalline-B Liquid Crystals
}

\section{Citation}

Hirth, J. P., Peter S. Pershan, J. Collett, E. Sirota, and L. B. Sorensen. 1984. Dislocation model for restacking phase transitions in crystalline-B liquid crystals. Physical Review Letters 53(5): 473-476.

\section{Published Version}

doi:10.1103/PhysRevLett.53.473

\section{Permanent link}

http://nrs.harvard.edu/urn-3:HUL.InstRepos:10361600

\section{Terms of Use}

This article was downloaded from Harvard University's DASH repository, and is made available under the terms and conditions applicable to Other Posted Material, as set forth at http:// nrs.harvard.edu/urn-3:HUL.InstRepos:dash.current.terms-of-use\#LAA

\section{Share Your Story}

The Harvard community has made this article openly available.

Please share how this access benefits you. Submit a story.

Accessibility 


\title{
Dislocation Model for Restacking Phase Transitions in Crystalline-B Liquid Crystals
}

\author{
J. P. Hirth \\ Department of Metallurgical Engineering, The Ohio State University, Columbus, Ohio 43210 \\ and \\ P. S. Pershan, J. Collett, ${ }^{(a)}$ E. Sirota, and L. B. Sorensen ${ }^{(b)}$ \\ Division of Applied Sciences, Harvard University, Cambridge, Massachusetts 02138
}

(Received 20 March 1984; revised manuscript received 14 June 1984)

\begin{abstract}
A dislocation-mediated model is presented for restacking phase transitions that have been observed in a variety of lamellar (liquid crystalline) systems. The model explains the existence of nonhexagonal crystalline (smectic) $-B$ phases in terms of dislocation-induced tilting of hexagonally packed layers. Ordered dislocation arrays explain both the symmetry and the amplitude of observed modulations. It is likely that the model will also be applicable to modulated lipid-water phases.
\end{abstract}

PACS numbers: $64.70 . \mathrm{Ew}, 61.30 .-\mathrm{v}, 81.30 . \mathrm{Kf}$

Current ideas concerning the types of order and phase transitions in two-dimensional systems ${ }^{1,2}$ have stimulated renewed theoretical interest in dislocation-mediated phase transitions in both two and three dimensions. ${ }^{3,4}$ Unfortunately, there are not many experimentally observed phase transitions that can be unambiguously interpreted in terms of dislocations. One of the more interesting properties of many liquid crystal materials is that on cooling they form hexagonal, or nearly hexagonal, layered crystals that exhibit subtle structural phase transitions within a small temperature range. ${ }^{5-7}$ In this Letter we demonstrate a dislocation model that explains most of the features of the structural phase transitions observed in three-dimensional samples of heptyloxybenzylidene-heptylaniline, $70.7 .^{7}$ Nearly all of the liquid crystalline systems that have phases with long-range in-plane positional order (i.e., smectic $B, G, H$, etc.) ${ }^{8,9}$ exhibit similar phase transitions. In most cases where there is a lowtemperature phase with tilted molecules, they are accompanied by an unexplained long-wavelength modulation that is similar to the modulation accompanying one of the principal phase transitions commonly observed in lipid-water mixtures. ${ }^{10}$ We believe the proposed model is generally applicable to all of the liquid crystal systems and may also be applicable to lipid-water systems. In the latter case, Chan and Webb interpreted the appearance of long-wavelength modulation in terms of a martensitic transformation. Although dislocation models for martensitic transformations have been discussed previously, the present specific model has new features that might well explain the lipid-water system and also be applicable to metals.

Between 69 and $63^{\circ} \mathrm{C}$ thick films of 70.7 form an hexagonal close packed (hcp) crystal with $A B A B$ stacking. Figure 1 (a) displays the $A, B$, and $C$ sites of a two-dimensional hexagonal lattice. At $63^{\circ} \mathrm{C}$ 70.7 undergoes a first-order transition in which the macroscopic symmetry changes from hcp to a modulated orthorhombic- $F$ phase. The $\mathrm{x}$-ray structure of the latter has been explained by assuming that the molecules at the $B$ sites move to the position marked by $X$ one layer below (or above) the $A$

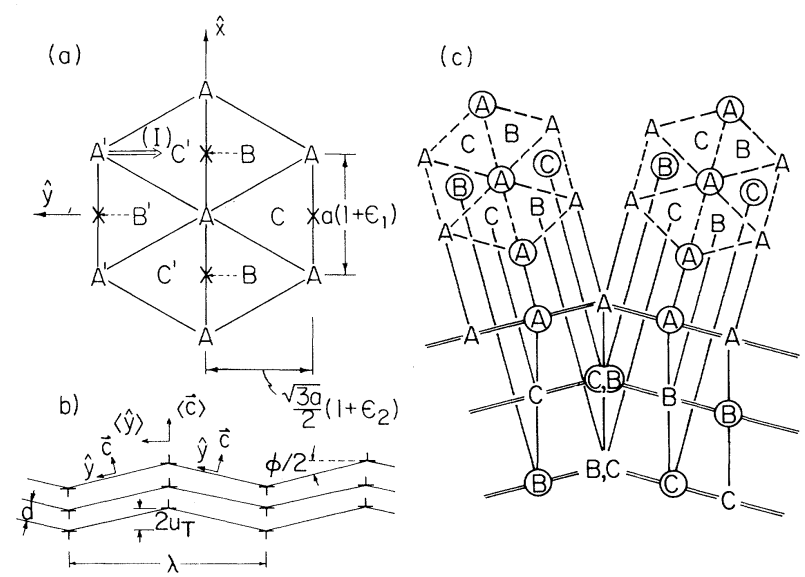

FIG. 1. (a) Hexagonal lattice sites $A, B$, and $C$. The hexagonal lattice with alternate layers in the $A$ and $B$ positions transform to orthorhombic- $F$ by displacing the $B$ positions to the $X$ position. Partial dislocations with Burgers vector (I) in every second layer transforms the hexagonal $A B A B$ to a hexagonal $A B C A B C$. The notation for in-plane strains $\epsilon_{1}$ and $\epsilon_{2}$ is shown. (b) A regular array of edge dislocations of opposite sign produces tilt boundaries as shown. (c) Projection of lattices that are locally hexagonal $A B C$ and $A C B$ onto tilted layers to obtain a modulated orthorhombic- $F$ lattice. Circle letters indicate equivalent $x$ coordinates. Viewed from opposite sides of the tilt boundary, single molecular sites in the interface are either $B$ or $C$. 
sites. ${ }^{7}$ Accompanying this is the appearance of sidebands on the $(0,0,1),(1,0, l)$ Bragg peaks with $l \neq 0$ due to a long-wavelength modulation, $\lambda \simeq 18.8 a$, with wave vector parallel to the $B X$ direction and displacements normal to the layers. For the $(1,0,1)$ Bragg peak the only observed sideband is the lowest order, with the intensity $\simeq 2 \%$ of the main peak. Shifts in the positions of the $(1,0, l),(0,1, l)$, and $(1,1, l)$ Bragg peaks imply strains of $\epsilon_{1}=-\epsilon_{2}$ $=0.001$.

The literature abounds with successful phase transformation models involving localized defects of either the shear type, the shuffle type, or a mixture of the two. ${ }^{11,12}$ The common feature is the assumption of spontaneously nucleated dislocation loops of small Burgers vector that grow and interact to produce a macroscopic transformation. In the present case, consider a partial dislocation in one layer of $A$ sites that transforms the crystal from $A B A B A B A B A$ to $A B C A C A C A C$ stacking. The Burgers vector (I) is shown in Fig. 1(a). ${ }^{13}$ Consider further that if a similar dislocation is repeated every second layer throughout the crystal the original lattice is converted to $A B C A B C A B C$. This is an obvious possibility for the molecule pentyloxybenzylidine-hexylaniline (50.6) that is only slightly different from 70.7. In the case of (50.6) there is a hexagonal phase with $A B C$ stacking between a hexagonal phase with $A B A B$ stacking and the orthorhombic- $F$ phase. ${ }^{14}$

If, however, we assume that in 70.7 the Burgers vector of each of the successive dislocations were parallel to one another, and that the dislocations interacted to be above one another the result would be a tilt boundary. Figure 1(b) illustrates a regular array of tilt boundaries of opposite sign. For 70.7 the layer thickness $d \simeq 6 a$. According to the Frank formula ${ }^{15}$ the angle $\phi / 2=\sin ^{-1}(a \sqrt{3} / 6 d)=0.048$ is exactly the correct tilt to put the sites $A^{\prime}, B^{\prime}$, and $C^{\prime}$ [Fig. 1(a)] in a common vertical plane as required for the orthorhombic- $F$.

Figure 1(c) illustrates the molecular positions. On the two sides of the tilt boundary the local packing is either hexagonal $A B C$ or $A C B$. To the right of the tilt boundary the transformation is as described above. To the left, however, the dislocations occur in the alternate layers and the transformation is from $A B A B A B$ to $A C B A C B$. However, as viewed along the average $\langle\bar{c}\rangle$ axis the molecules form the face-centered rectangle of the orthorhombic- $F$ phase.

A model to explain the formation of a regular dislocation array is illustrated in Figs. 2(a) and 2(b). With the assumption of spontaneous nucleation of a

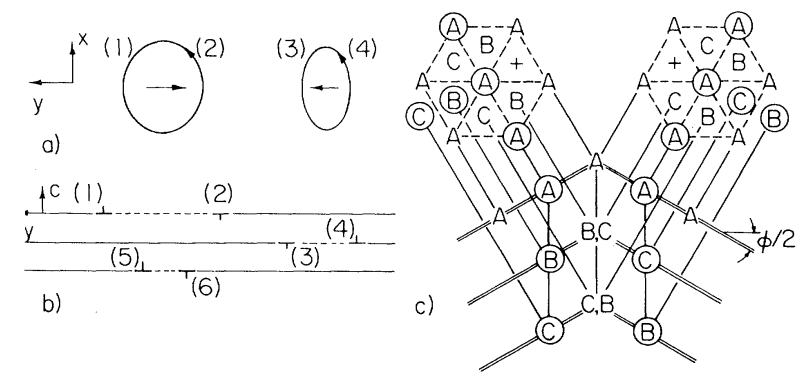

FIG. 2. (a) Top view of two dislocation loops (1)-(2) and (3)-(4). The arrows inside the loops indicate Burgers vectors and those on the loops the senses. (b) Side view of dislocations loops whose elastic interactions favor mutual formation. (c) Projection of a lattice that is hexagonal $A B C$ onto tilted layers to obtain a modulated hexagonal $A A A$ lattice. The plus indicates a missing molecule due to the interposition of dislocations.

single dislocation loop (1)-(2) of the type discussed above, the elastic-strain field provides a loop-loop elastic interaction favoring nucleation of the loop (5)-(6) two planes directly above or below. ${ }^{16}$ These two will then produce a field favoring nucleation of a (3)-(4), one plane removed from each, displaced laterally, and of opposite sign. Successive loop nucleations of this kind produce the edge dislocation tilt walls of Fig. 1(b). The screw portions of the loops, with lines parallel to the $y$ direction could also meet and interact to form an array. Such an array, however, would not be a low-energy one (a crossed-grid of screw dislocations is required for a stable, low-angle twist boundary). ${ }^{12}$ The attendant repulsive interactions among screw segments would tend to cause them to slip out of the crystal, leaving the edge array of Fig. 1(b).

To evaluate the quantitative predictions of the model note that the values quoted above for the strains $\epsilon_{1}$ and $\epsilon_{2}$ were obtained from shifts in the positions of the Bragg peaks. If we define $\left(\epsilon_{1}^{T}, \epsilon_{2}^{T}\right)$ to be the strains measured along the local $\hat{x}, \hat{y}$ axis, then one can show that the strains $\left(\epsilon_{2}, \epsilon_{2}\right)$ measured relative to the average $\hat{x},\langle\hat{y}\rangle$ axis are $\epsilon_{1}=\epsilon_{1}^{T}$, $\epsilon_{2}=\epsilon_{2}^{T}-\phi^{2} / 8$. Under the assumption that the transformation is accompanied by a simple area dilation in the local $\hat{x}-\hat{y}$ smectic layers, one obtains $\epsilon_{1}^{T}=\epsilon_{2}^{T}=0.001$ and $\phi / 2=(0.004)^{1 / 2}=0.063$. This should be compared with the value of 0.048 obtained from the Frank formula under the implicit assumption that $\left(\epsilon_{1}^{T}, \epsilon_{2}^{T}\right)=0$. With the assumption of the triangular modulation shown in Fig. 1, the predicted ratio of sideband to $(1,0,1)$ peak intensity $\left(8 u_{T} / \pi d\right)^{2} \approx(\phi \lambda / \pi d)^{2} \approx 0.016$. The measured ratio was $\simeq 0.02$. Although we are not able to predict 
the value of $\lambda$ this quantitative agreement strongly supports the model.

Further qualitative support for the model is the above-mentioned hexagonal phase with $A B C$ stacking that occurs in 50.6 between the hexagonal $A B A B$ phase and the orthorhombic- $F$ (48 to $\left.43.8^{\circ} \mathrm{C}\right)$. The Bragg peaks that occur in this phase at $\left(1,0, \pm \frac{2}{3}\right)$ and $\left(1,0, \pm \frac{1}{3}\right)$ are accompanied by diffuse sidebands cylindrically distributed about the peak. ${ }^{14}$ The transition at $48^{\circ} \mathrm{C}$ produces layers with $A B C$ stacking; however, according to the model the accompanying dislocations have not condensed into the regular array. This happens in 50.6 at $43.8^{\circ} \mathrm{C}$ where one observes a modulated othorhombic- $F$ phase identical to that in 70.7.

At $60.1^{\circ} \mathrm{C}$ thick films of 70.7 undergo a second transition from the orthorhombic- $F$ to a monoclinic phase. The $\mathrm{x}$-ray data for this phase are consistent with the assumption that the molecules located at the $X$ position in Fig. 1(a) moved along the line $X A$. Following a small sudden displacement at $60.1^{\circ} \mathrm{C}$ the molecules move continuously with decreasing temperature until at $59.75^{\circ} \mathrm{C}$ there is a second small displacement that places this layer directly under the $A$ position in the adjacent layer. These effects can also be explained by an extension of the previous model. For example, Fig. 2(c) displays a structure similar to that of Fig. 1(c) except that it has a modulated $A A A$ structure. The tilt angle $\phi / 2$ required to produce this structure, $\tan ^{-1}(\sqrt{3} a / 3 d)$, is exactly twice the tilt required to produce the orthorhombic- $F$ structure. One way to produce this starting from the orthorhombic phase depicted in Fig. 1(c) is illustrated in Fig. 3. Starting from a structure that is locally $A B C A B C A$ (orthorhombic-
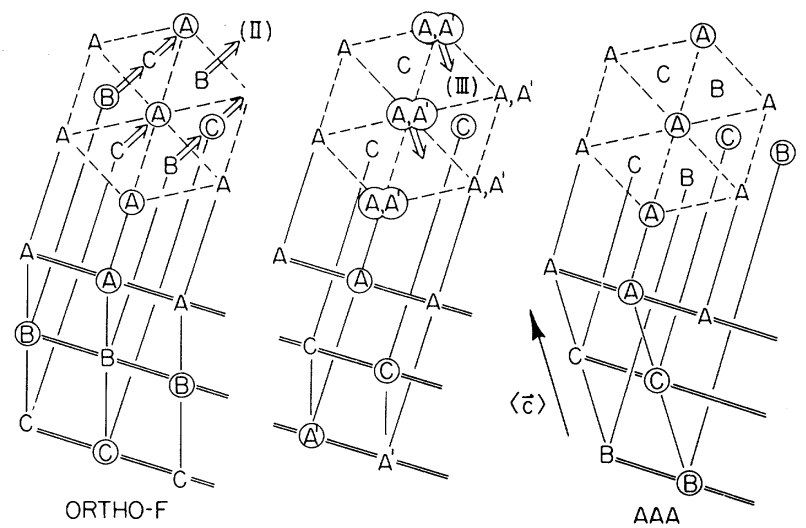

FIG. 3. Intermediate steps in the dislocation-induced transition from the orthorhombic- $F$ to the hexagonal$A A A$ phase.
F) a partial dislocation in the second layer (of the same type as invoked previously) with Burgers vector (II) converts the structure to the $A C A B C A B$ structure when in the center panel. A second dislocation, either the same type (II) or of the type (III) in the third layer converts this to $A C B C A B C$. If this sequence is repeated one obtains an $A C B A C$ $B A C B$ structure with an increase in the Frank tilt by $\Delta(\phi / 2)=\tan ^{-1}(\sqrt{3} a / 6 d)$. This is exactly the correct value to be added to the orthorhombic- $F$ phase to obtain the structure in Fig. 2(c).

The monoclinic phase that separates the orthorhombic- $F$ and the hexagonal $A A A$ would then be an intermediate phase for which there were some statistical distribution in the number of type II and III dislocations to produce a domain with a component of tilt along the $x$ direction. Obviously the sequence could have begun with a type III, rather than type II, dislocation. According to this model the maximum component of tilt due to a coherent stacking of only type (II) dislocations would be $\phi_{y} / 2 \simeq \tan ^{-1}(a / 2 d) \simeq 0.083$ about the $\hat{y}$ axis and $\left(\phi_{x} / 2\right) \simeq \tan ^{-1}(\sqrt{3} a / 3 d)=0.096$, about the $\hat{x}$ axis. Assuming the intralayer strain remains isotropic (e.g., $\epsilon_{1}^{T}=\epsilon_{2}^{T}$ ) the predicted anisotropy in the reciprocal lattice vectors (see Fig. 3 of Ref. 7) is $\frac{3}{8}\left[\left(\phi_{x} / 2\right)^{2}-\left(\phi_{y} / 2\right)^{2}\right]=0.00086$. Although this is approximately three times larger than the observed splitting in the monoclinic phase the actual splitting is a very delicate function of not only the distribution of (II) and (III) dislocations but also of their physical distribution within the domains. The observed anisotropies could also be reduced by allowing $\epsilon_{1}^{T} \neq \epsilon_{2}^{T}$. The more significant feature is that it provides a rational explanation for the observed reduction in the splitting.

In conclusion, we have presented a dislocation model for restacking transitions observed in bulk phases of crystalline- $B$ liquid crystals. Features of these transitions appear similar to phase transitions in other systems, such as lipid-water mixtures, which have been widely studied and it is likely that the model here is relevant to those systems. The model also predicts that the orthorhombic- $F$ to hexagonal- $A B C$ transition in 50.6 should be an example of the crystallization of dislocations. Further experimental studies of this transition should be carried out with this in mind. The model is sufficiently specific that one might hope to develop a molecular model for the forces that generate the dislocations. For example, if these transitions are driven by a mechanism that causes a shearing of the original unit cell, the model illustrates how a set of minimum molecular displacements can accommo- 
date both the shear and the local molecular packing.

Finally, we do not believe there is a direct relation between the present model and the tilted hexatic phases that appear in thin films of 70.7. ${ }^{17}$ The large molecular tilt $\left(\simeq 24^{\circ}\right)$ is indicative of a separate thermodynamic phase that is probably stabilized by surface effects. Similarly, since the dislocations in the present model run parallel to the smectic layers they are not the same as dislocations normal to the layers that are commonly invoked to discuss two-dimensional melting. ${ }^{1,2}$

The research reported here was supported in part by the National Science Foundation under Grants No. DMR-82-12189 and No. DMR-80-20247. In addition one of us (J.P.H.) would like to acknowledge support from both Ohio State University and the Harvard Materials Research Laboratory while on sabbatical leave. Conversations with both D. Nelson and B. Halperin are also gratefully acknowledged.

\footnotetext{
(a) Present address: IBM Research Laboratory, Yorktown Heights, N.Y. 10598.

(b) Present address: Department of Physics, FM-15, University of Washington, Seattle, Wash. 98195.

1B. I. Halperin and D. R. Nelson, Phys. Rev. Lett. 41, 121 (1978). D. R. Nelson and B. I. Halperin, Phys. Rev. B 19, 2456 (1979).

${ }^{2}$ A. P. Young, Phys. Rev. B 19, 1855 (1979).

${ }^{3}$ C. Deutsch and S. Doniach, Phys. Rev. B 29, 2724
}

(1984)

${ }^{4}$ H. Kleinert, Phys. Lett. 96A, 302 (1983).

${ }^{5}$ A. J. Leadbetter, M. A. Mazid, B. A. Kelley, J. Goodby, and G. W. Gray, Phys. Rev. Lett. 43, 630 (1979).

${ }^{6} \mathrm{~J}$. Doucet and A. M. Levelut, J. Phys. (Paris) 38, 1163 (1977).

${ }^{7}$ J. Collett, L. B. Sorensen, P. S. Pershan, J. D. Litster, R. J. Birgeneau, and J. Als-Nielsen, Phys. Rev. Lett. 49, 553 (1982).

${ }^{8}$ A. J. Leadbetter, M. A. Mazid, and R. M. Richardson, in Liquid Crystals, edited by S. Chandrasekhar (Cambridge Univ. Press, London, 1980), pp. 65-79.

${ }^{9}$ P. A. C. Gane, A. J. Leadbetter, P. A. Tucker, G. W. Gray, and A. R. Tajbaksh, J. Chem. Phys. 77, 6215 (1982).

10W. K. Chan and W. W. Webb, Phys. Rev. Lett. 46, 39 (1981). See also Refs. 6-9, and J. W. Christian and A. G. Crocker, in Dislocations of Solids, edited by F. R. N. Nabarro (North-Holland, Amsterdam, 1980), Vol. 3, p. 165, and J. P. Hirth and J. Lothe, Theory of Dislocations (Wiley, New York, 1982), p. 707.

${ }^{11}$ Christian and Crocker, Ref. 10

${ }^{12}$ Hirth and Lothe, Ref. 10.

${ }^{13}$ Similar dislocations appear in computer simulations of dislocation mediated melting carried out by R. M. J. Cotterill, W. Damgaard Kristensen, and E. J. Jensen, Philos. Mag. 30, 245 (1974).

${ }^{14}$ Jeffrey Allen Collett, "X-Ray Scattering Study of Liquid Crystal Thin Films," Ph.D. thesis, Harvard University, June 1983 (unpublished).

${ }^{15}$ F. C. Frank, Discuss. Faraday Soc. 25, 19 (1958).

${ }^{16}$ Hirth and Lothe, Ref. 10, p. 733.

${ }^{17}$ Jeffrey Collett, P. S. Pershan, Eric B. Sirota, and L. B. Sorensen, Phys. Rev. Lett. 52, 356 (1984). 